\title{
Possible quantum kinematics. II. Non-minimal case
}

\author{
N.A. Gromov \\ Department of Mathematics, \\ Komi Science Center UrD RAS \\ Chernova st., 3a, Syktyvkar, 167982, Russia \\ E-mail: gromov@dm.komisc.ru
}

\begin{abstract}
The quantum analogs of the $N$-dimensional Cayley-Klein spaces with different combinations of quantum and Cayley-Klein structures are described for non-minimal multipliers, which include the first and the second powers of contraction parameters in the transformation of deformation parameter. The noncommutative analogs of $(N-1)$-dimensional constant curvature spaces are introduced. Part of these spaces for $N=5$ are interpreted as the noncommutative analogs of $(1+3)$ space-time models. As a result the wide variety of the quantum deformations of realistic kinematics are suggested.

PACS: 02.40.Gh, 02.40.Yy, 04.20.Gz
\end{abstract}

\section{Introduction}

Space-time is a fundamental conception that underlines the most significant physical theories. Therefore the analysis of a possible space-time models has the fundamental meaning for physics. In the previous papers [1, [2] the noncommutative analogs of the possible commutative kinematics [3] was constructed starting from the mathematical theory of quantum groups and quantum vector spaces [4]. Cayley-Klein scheme of contractions and analytical continuations was applied to the five dimensional q-Euclidean space. Different combinations of quantum structure and Cayley-Klein scheme was described with the help of permutations $\sigma$.

The transformation of the deformation parameter $z=J v$ under contraction is added in the quantum case as compared with the commutative one. The analysis in [1, [2] was confined to the minimal multiplier $J$, which has the first power multiplication of contraction parameters. This restriction imply that for certain combinations of quantum structure and Cayley-Klein scheme kinematics contractions do not exist. In order to all Cayley-Klein 
contractions was possible for all permutations $\sigma$ it is necessary to regard non-minimal multiplier $J$, which include the first and the second powers of contraction parameters. In this paper we find non-minimal multipliers for the general quantum Cayley-Klein spaces (Sec. 2) and describe noncommutative four-dimensional space-time models (Sec. 3).

\section{Quantum Cayley-Klein spaces}

It was shown in the previous paper [1] that the quantum $\mathrm{N}$-dimensional Cayley-Klein space $O_{v}^{N}(j ; \sigma ; \mathbf{C})$ is characterized by the following commutation relations of the Cartesian generators

$$
\begin{gathered}
\xi_{\sigma_{k}} \xi_{\sigma_{m}}=\xi_{\sigma_{m}} \xi_{\sigma_{k}} \cosh J v-i \xi_{\sigma_{m}} \xi_{\sigma_{k^{\prime}}} \frac{\left(1, \sigma_{k^{\prime}}\right)}{\left(1, \sigma_{k}\right)} \sinh J v, k<m<k^{\prime}, k \neq m^{\prime}, \\
\xi_{\sigma_{k}} \xi_{\sigma_{m}}=\xi_{\sigma_{m}} \xi_{\sigma_{k}} \cosh J v-i \xi_{\sigma_{m^{\prime}}} \xi_{\sigma_{k}} \frac{\left(1, \sigma_{m^{\prime}}\right)}{\left(1, \sigma_{m}\right)} \sinh J v, m^{\prime}<k<m, k \neq m^{\prime}, \\
{\left[\xi_{\sigma_{k}}, \xi_{\sigma_{k^{\prime}}}\right]=2 i \epsilon \sinh \left(\frac{J v}{2}\right)(\cosh J v)^{n-k} \xi_{\sigma_{n+1}}^{2} \frac{\left(1, \sigma_{n+1}\right)^{2}}{\left(1, \sigma_{k}\right)\left(1, \sigma_{k^{\prime}}\right)}+} \\
+i \frac{\sinh (J v)}{(\cosh J v)^{k+1}\left(1, \sigma_{k}\right)\left(1, \sigma_{k^{\prime}}\right)} \sum_{m=k+1}^{n}(\cosh J v)^{m}\left(\left(1, \sigma_{m}\right)^{2} \xi_{\sigma_{m}}^{2}+\left(1, \sigma_{m^{\prime}}\right)^{2} \xi_{\sigma_{m^{\prime}}}^{2}\right),
\end{gathered}
$$

where $k, m=1,2, \ldots, n, N=2 n+1$ or $N=2 n, k^{\prime}=N+1-k$, permutation $\sigma=\left(\sigma_{1}, \ldots, \sigma_{N}\right)$ describes definite combination of the quantum group structure and Cayley-Klein scheme of group contraction. The invariant form under the coaction of the corresponding quantum orthogonal group on the Cayley-Klein space $O_{v}^{N}(j ; \sigma ; \mathbf{C})$ is written as

$$
\begin{gathered}
\operatorname{inv}(j)=\cosh \left(J v \rho_{1}\right)\left(\epsilon\left(1, \sigma_{n+1}\right)^{2} \xi_{\sigma_{n+1}}^{2} \frac{(\cosh J v)^{n}}{\cosh (J v / 2)}+\right. \\
\left.+\sum_{k=1}^{n}\left(\left(1, \sigma_{k}\right)^{2} \xi_{\sigma_{k}}^{2}+\left(1, \sigma_{k^{\prime}}\right)^{2} \xi_{\sigma_{k^{\prime}}}^{2}\right)(\cosh J v)^{k-1}\right) .
\end{gathered}
$$

The multiplier $J$ in the transformation $z=J v$ of the deformation parameter need be chosen in such a way that all indefinite relations in commutators, which appear under nilpotent values of the contraction parameters are canceled. As far as multipliers $\left(1, \sigma_{k}\right)$ and $\left(1, \sigma_{k^{\prime}}\right)$ enter symmetrically into the commutators (11),(2), we can put $\sigma_{k}<\sigma_{k^{\prime}}$ without loss 
of generality. Then indefinite relations in commutators (11) take the form $\left(1, \sigma_{k}\right)\left(1, \sigma_{k^{\prime}}\right)^{-1}=\left(\sigma_{k}, \sigma_{k^{\prime}}\right)$, where $k=1,2, \ldots, n$ for $N=2 n+1$ and $k=1,2, \ldots, n-1$ for $N=2 n$. They are eliminated by the multiplier

$$
J_{0}=\bigcup_{k=1}^{n}\left(\sigma_{k}, \sigma_{k^{\prime}}\right)
$$

It has the first power multiplication of contraction parameters and is the minimal multiplier, which guarantees the existence of the Hopf algebra structure for the associated quantum group $S O_{v}(N ; j ; \sigma)$. The analysis in the previous paper [1] was confined to this minimal case.

The "union" of two multipliers is understood as the multiplication of all parameters $j_{k}$, which occur at least in one multiplier and the power of $j_{k}$ in the "union" is equal to its maximal power in both multipliers, for example, $\left(j_{1} j_{2}^{2}\right) \cup\left(j_{2} j_{3}\right)=j_{1} j_{2}^{2} j_{3}$.

If we take into account indefinite relations in commutators (2), then we come to the non-minimal multiplier $J$, which consist of contraction parameters in the first and the second powers. The indefinite relations in commutators (2) have the form

$$
\frac{\sum_{m=k+1}^{n}\left[\left(1, \sigma_{m}\right)^{2}+\left(1, \sigma_{m^{\prime}}\right)^{2}\right]}{\left(1, \sigma_{k}\right)\left(1, \sigma_{k^{\prime}}\right)}=\frac{\sum_{m=k+1}^{n}\left(1, \sigma_{m}\right)^{2}}{\left(1, \sigma_{k}\right)^{2}\left(\sigma_{k}, \sigma_{k^{\prime}}\right)}
$$

$k=1, \ldots, n-1$ for even $N=2 n$ and

$$
\frac{\left(1, \sigma_{n+1}\right)+\sum_{m=k+1}^{n}\left[\left(1, \sigma_{m}\right)^{2}+\left(1, \sigma_{m^{\prime}}\right)^{2}\right]}{\left(1, \sigma_{k}\right)\left(1, \sigma_{k^{\prime}}\right)}=\frac{\left(1, \sigma_{n+1}\right)+\sum_{m=k+1}^{n}\left(1, \sigma_{m}\right)^{2}}{\left(1, \sigma_{k}\right)^{2}\left(\sigma_{k}, \sigma_{k^{\prime}}\right)},
$$

$k=1, \ldots, n$ for odd $N=2 n+1$. Let us introduce numbers

$$
i_{k}=\min \left\{\sigma_{k+1}, \ldots, \sigma_{n}\right\},
$$

then $k$ th expression in (5) or (6) is equal to

$$
\frac{\left(1, i_{k}\right)^{2}}{\left(1, \sigma_{k}\right)^{2}\left(\sigma_{k}, \sigma_{k^{\prime}}\right)}=\left\{\begin{array}{cc}
\left(i_{k}, \sigma_{k}\right)^{-2}\left(\sigma_{k}, \sigma_{k^{\prime}}\right)^{-1}, & i_{k}<\sigma_{k} \\
\left(\sigma_{k}, i_{k}\right)\left(i_{k}, \sigma_{k^{\prime}}\right)^{-1}, & \sigma_{k}<i_{k}<\sigma_{k^{\prime}} \\
\left(\sigma_{k}, \sigma_{k^{\prime}}\right)\left(\sigma_{k^{\prime}}, i_{k}\right)^{2}, & i_{k}>\sigma_{k^{\prime}}
\end{array}\right.
$$

and compensative multiplier for this expression is as follows

$$
J_{1}^{(k)}=\left\{\begin{array}{cc}
\left(i_{k}, \sigma_{k}\right)^{2}\left(\sigma_{k}, \sigma_{k^{\prime}}\right), & i_{k}<\sigma_{k} \\
\left(i_{k}, \sigma_{k^{\prime}}\right), & \sigma_{k}<i_{k}<\sigma_{k^{\prime}} \\
1, & i_{k}>\sigma_{k^{\prime}}
\end{array}\right.
$$


For all expressions in (5) or (6) compensative multiplier $J_{1}$ is obtained by the union $J_{1}=\bigcup_{k} J_{1}^{(k)}$. Therefore the non-minimal multiplier $J$ in the transformation $z=J v$ of the deformation parameter is given by

$$
J=J_{0} \bigcup J_{1}=J_{0} \bigcup_{k} J_{1}^{(k)}
$$

and include the first and the second powers of contraction parameters.

The quantum orthogonal Cayley-Klein sphere $S_{v}^{(N-1)}(j ; \sigma)$ is obtained as the quotient of $O_{v}^{N}(j ; \sigma)$ by $i n v(j)=1$. The quantum analogs of the intrinsic Beltrami coordinates on this quantum sphere are given by the sets of independent right or left generators

$$
r_{\sigma_{i}-1}=\xi_{\sigma_{i}} \xi_{1}^{-1}, \quad \hat{r}_{\sigma_{i}-1}=\xi_{1}^{-1} \xi_{\sigma_{i}}, \quad i=1, \ldots, N, \quad i \neq k, \quad \sigma_{k}=1
$$

Quantum kinematics are realized as quantum orthogonal sphere for $N=5$ and certain values of contraction parameters.

\section{Quantum kinematics}

Quantum vector spaces $O_{v}^{5}(\mathbf{j} ; \sigma)$ are generated by $\xi_{\sigma_{l}}, l=1, \ldots, 5$ with commutation relations $(k=2,3,4)$

$$
\begin{gathered}
\xi_{\sigma_{1}} \xi_{\sigma_{k}}=\xi_{\sigma_{k}} \xi_{\sigma_{1}} \cosh (J v)-i \xi_{\sigma_{k}} \xi_{\sigma_{5}} \frac{\left(1, \sigma_{5}\right)}{\left(1, \sigma_{1}\right)} \sinh (J v), \\
\xi_{\sigma_{k}} \xi_{\sigma_{5}}=\xi_{\sigma_{5}} \xi_{\sigma_{k}} \cosh (J v)-i \xi_{\sigma_{1}} \xi_{\sigma_{k}} \frac{\left(1, \sigma_{1}\right)}{\left(1, \sigma_{5}\right)} \sinh (J v), \\
\xi_{\sigma_{2}} \xi_{\sigma_{3}}=\xi_{\sigma_{3}} \xi_{\sigma_{2}} \cosh (J v)-i \xi_{\sigma_{3}} \xi_{\sigma_{4}} \frac{\left(1, \sigma_{4}\right)}{\left(1, \sigma_{2}\right)} \sinh (J v), \\
\xi_{\sigma_{3}} \xi_{\sigma_{4}}=\xi_{\sigma_{4}} \xi_{\sigma_{3}} \cosh (J v)-i \xi_{\sigma_{2}} \xi_{\sigma_{3}} \frac{\left(1, \sigma_{2}\right)}{\left(1, \sigma_{4}\right)} \sinh (J v), \\
{\left[\xi_{\sigma_{2}}, \xi_{\sigma_{4}}\right]=2 i \xi_{\sigma_{3}}^{2} \frac{\left(1, \sigma_{3}\right)^{2}}{\left(1, \sigma_{2}\right)\left(1, \sigma_{4}\right)} \sinh (J v / 2),} \\
{\left[\xi_{\sigma_{1}}, \xi_{\sigma_{5}}\right]=2 i\left(\xi_{\sigma_{3}}^{2}\left(1, \sigma_{3}\right)^{2} \cosh (J v)+\left(\xi_{\sigma_{2}}^{2}\left(1, \sigma_{2}\right)^{2}+\right.\right.}
\end{gathered}
$$




$$
\left.\left.+\xi_{\sigma_{4}}^{2}\left(1, \sigma_{4}\right)^{2}\right) \cosh (J v / 2)\right) \frac{\sinh (J v / 2)}{\left(1, \sigma_{1}\right)\left(1, \sigma_{5}\right)} .
$$

By the analysis of the multiplier (8) for $N=5$ and commutation relations (10) of the quantum space generators we have find three different non-minimal $J$, namely $J=j_{1}^{2} j_{2}, J=j_{1} j_{2}^{2}$ and $J=j_{1}^{2} j_{2}^{2}$. In the first two cases all different permutations give in result equivalent kinematics, so we can regard only one permutation, say $\hat{\sigma}=(2,1,3,4,5)$ for $J=j_{1}^{2} j_{2}$ and $\check{\sigma}=(1,3,2,4,5)$ for $J=j_{1} j_{2}^{2}$. In the case of $J=j_{1}^{2} j_{2}^{2}$ there are four nonequivalent quantum kinematics corresponding to the following permutations: $\tilde{\sigma}=$ $(2,3,1,4,5), \sigma_{I}=(3,1,5,2,4), \sigma_{I I}=(3,1,2,4,5), \sigma_{I I I}=(3,2,1,4,5)$.

In order to clarify the relation with the standard Inön $\ddot{u}-$ Wigner contraction procedure [5], the mathematical parameter $j_{1}$ is replaced by the physical one $\tilde{j}_{1} T^{-1}$, and the parameter $j_{2}$ is replaced by $i c^{-1}$, where the standard de Sitter kinematics $S_{4}^{(-)}$with constant negative curvature is realized for $\tilde{j}_{1}=i$ and anti de Sitter kinematics $S_{4}^{(+)}$with positive curvature is obtained for $\tilde{j}_{1}=1$.

The limit $T \rightarrow \infty$ corresponds to the contraction $j_{1}=\iota_{1}$, and the limit $c \rightarrow \infty$ corresponds to $j_{2}=\iota_{2}$. The parameter $T$ is interpreted as the curvature radius and has the physical dimension of time $[T]=[$ time], the parameter $c$ is the light velocity $[c]=\left[\right.$ length] $[\text { time }]^{-1}$. As far as argument $J v$ of hyperbolic function must be dimensionless, then the physical dimension of deformation parameter is equal to those of $J$, i.e. $[v]=[J]$. For $J=j_{1}^{2} j_{2}$ we obtain $[v]=\left[c T^{2}\right]=[$ length $][$ time $]$, for $J=j_{1} j_{2}^{2}$ we have $[v]=\left[c^{2} T\right]=$ $[\text { length }]^{2}[\text { time }]^{-1}=$ [length $]\left[\right.$ velocity] and for $J=j_{1}^{2} j_{2}^{2}$ the physical dimension of the deformation parameter is given by $[v]=\left[c^{2} T^{2}\right]=[\text { length }]^{2}$.

The generator $\xi_{1}$ does not commute with others, therefore it is convenient to introduce right and left time $t=\xi_{2} \xi_{1}^{-1}, \hat{t}=\xi_{1}^{-1} \xi_{2}$ and space $r_{k}=\xi_{k+2} \xi_{1}^{-1}, \hat{r}_{k}=\xi_{1}^{-1} \xi_{k+2}, k=1,2,3$, generators. The reason for this definition is the simplification of expressions for commutation relations of quantum kinematics. It is possible to use only, say, right generators, but its commutators are cumbersome in the case of the (anti) de Sitter kinematics.

\subsection{Quantum (anti) de Sitter kinematics}

For $\hat{\sigma}=(2,1,3,4,5)$ the mathematical multiplier $J=j_{1}^{2} j_{2}$ is rewritten in the

form $J=i \frac{\tilde{j}_{1}^{2}}{c T^{2}}=i \hat{J}$. Commutation relations of space and time generators of 
the (anti) de Sitter kinematics are

$$
\begin{gathered}
S_{v}^{4( \pm)}(\hat{\sigma})=\left\{t, \mathbf{r} \mid \hat{t} r_{p}=\hat{r}_{p}\left(t \cos \hat{J} v+i r_{3} \frac{1}{c} \sin \hat{J} v\right),\right. \\
\hat{t} r_{3}-\hat{r}_{3} t=2 i\left(-\frac{\tilde{j}_{1}^{2}}{c^{2} T^{2}} \hat{r}_{1} r_{1} \cos \hat{J} v+\left(1-\frac{\tilde{j}_{1}^{2}}{c^{2} T^{2}} \hat{r}_{2} r_{2}\right) \cos \frac{\hat{J} v}{2}\right) \frac{c T^{2}}{\tilde{j}_{1}^{2}} \sin \frac{\hat{J} v}{2}, \\
\left.\hat{r}_{p} r_{3}=\left(\hat{r}_{3} \cos \hat{J} v-i \hat{t} c \sin \hat{J} v\right) r_{p}, \quad \hat{r}_{1} r_{2}=\left(\hat{r}_{2} \cos \hat{J} v-i \hat{t} \frac{c T}{\tilde{j}_{1}} \sin \hat{J} v\right) r_{1}\right\} .
\end{gathered}
$$

The right and left generators are connected as follows

$$
\begin{gathered}
\hat{t}=t \cos \hat{J} v+i r_{3} \frac{1}{c} \sin \hat{J} v, \quad r_{1}=\hat{r}_{1}\left(\cos \hat{J} v+i r_{2} \frac{\tilde{j}_{1}}{c T} \sin \hat{J} v\right), \\
\hat{r}_{2}-r_{2}=2 i \frac{\tilde{j_{1}}}{c T} \hat{r}_{1} r_{1} \sin \frac{\hat{J} v}{2}, \quad \hat{r}_{3}=r_{3} \cos \hat{J} v+i t c \sin \hat{J} v
\end{gathered}
$$

For $\check{\sigma}=(1,3,2,4,5)$ the mathematical multiplier $J=j_{1} j_{2}^{2}$ is rewritten in the form $J=-\frac{\tilde{j_{1}}}{c^{2} T}=-\check{J}$. Commutation relations of space and time generators of the (anti) de Sitter kinematics are

$$
\begin{gathered}
S_{v}^{4( \pm)}(\check{\sigma})=\left\{t, \mathbf{r} \mid \hat{r_{1}} t=\hat{t}\left(r_{1} \cosh \check{J} v+i r_{2} \sinh \check{J} v\right),\right. \\
\hat{t} r_{2}=\left(\hat{r}_{2} \cosh \check{J} v+i \hat{r}_{1} \sinh \check{J} v\right) t, \quad \hat{t} r_{3}=\left(\hat{r}_{3} \cosh \check{J} v+\frac{c T}{\tilde{j}_{1}} \sinh \check{J} v\right) t, \\
\left.\hat{r_{1}} r_{2}-r_{2} \hat{r}_{1}=2 i \hat{t} t c^{2} \sinh \frac{\check{J} v}{2}, \quad \hat{r}_{p} r_{3}=\left(\hat{r}_{3} \cosh \check{J} v+\frac{c T}{\tilde{j}_{1}} \sinh \check{J} v\right) r_{p}\right\} .
\end{gathered}
$$

The right and left generators are connected as follows

$$
\begin{aligned}
& t=\hat{t}\left(\cosh \check{J} v-\frac{\tilde{j_{1}}}{c T} r_{3} \sinh \check{J} v\right), r_{p}=\hat{r}_{p}\left(\cosh \check{J} v-\frac{\tilde{j}_{1}}{c T} r_{3} \sinh \check{J} v\right), \\
& \hat{r}_{3}-r_{3}=\frac{2}{\tilde{j}_{1}} c T\left(\hat{t} t \cosh \check{J} v-\frac{\tilde{j}_{1}^{2}}{c^{2} T^{2}}\left(\hat{r}_{1} r_{1}+\hat{r}_{2} r_{2}\right) \cosh \frac{\check{J} v}{2}\right) \sinh \frac{\check{J} v}{2} .
\end{aligned}
$$


For $\tilde{\sigma}=(2,3,1,4,5)$ the mathematical multiplier $J=j_{1}^{2} j_{2}^{2}$ is rewritten in the form $J=-\frac{\tilde{j}_{1}^{2}}{c^{2} T^{2}}=-\tilde{J}$. Commutation relations of space and time generators of the (anti) de Sitter kinematics are

$$
\begin{gathered}
S_{v}^{4( \pm)}(\tilde{\sigma})=\left\{t, \mathbf{r} \mid \hat{t} r_{p}=\hat{r}_{p}\left(t \cosh \tilde{J} v-r_{3} \frac{1}{c} \sinh \tilde{J} v\right)\right. \\
\hat{t r}_{3}-\hat{r}_{3} t=-2\left[\cosh \tilde{J} v-\frac{\tilde{j}_{1}^{2}}{c^{2} T^{2}}\left(\hat{r}_{1} r_{1}+\hat{r}_{2} r_{2}\right) \cosh \frac{\tilde{J} v}{2}\right] \frac{c T^{2}}{\tilde{j}_{1}^{2}} \sinh \frac{\tilde{J} v}{2}, \\
\left.\hat{r}_{p} r_{3}=\left(\hat{r}_{3} \cosh \tilde{J} v+\hat{t} c \sinh \tilde{J} v\right) r_{p}, \quad \hat{r}_{1} r_{2}-\hat{r}_{2} r_{1}=-2 i \frac{c^{2} T^{2}}{\tilde{j}_{1}^{2}} \sinh \frac{\tilde{J} v}{2}\right\}
\end{gathered}
$$

where $k=1,2,3, p=1,2$. The left and right generators are connected by the relations

$$
\begin{aligned}
& \hat{t}=t \cosh \tilde{J} v-r_{3} \frac{1}{c} \sinh \tilde{J} v, \quad \hat{r}_{1}=r_{1} \cosh \tilde{J} v+i r_{2} \sinh \tilde{J} v \\
& \hat{r}_{2}=r_{2} \cosh \tilde{J} v-i r_{1} \sinh \tilde{J} v, \quad \hat{r}_{3}=r_{3} \cosh \tilde{J} v-t c \sinh \tilde{J} v .
\end{aligned}
$$

For $\sigma_{I}=(3,1,5,2,4)$ commutation relations of space and time generators of the (anti) de Sitter kinematics are

$$
\begin{gathered}
S_{v}^{4( \pm)}\left(\sigma_{I}\right)=\left\{t, \mathbf{r} \mid \hat{r}_{1} t=\hat{t}\left(r_{1} \cosh \tilde{J} v+i r_{2} \sinh \tilde{J} v\right),\right. \\
\hat{t r}_{2}=\left(\hat{r}_{2} \cosh \tilde{J} v+i \hat{r}_{1} \sinh \tilde{J} v\right), \hat{r}_{3} t=\left(\hat{t} \cosh \tilde{J} v+i \frac{T}{\tilde{j}_{1}} \sinh \tilde{J} v\right) r_{3}, \\
\hat{r}_{1} r_{3}=\hat{r}_{3}\left(r_{1} \cosh \tilde{J} v+i r_{2} \sinh \tilde{J} v\right), \hat{r}_{3} r_{2}=\left(\hat{r}_{2} \cosh \tilde{J} v+i \hat{r}_{1} \sinh \tilde{J} v\right) r_{3}, \\
\left.\hat{r}_{1} r_{2}-\hat{r}_{2} r_{1}=2 i\left[-\frac{\tilde{j}_{1}^{2}}{c^{2} T^{2}} \hat{r}_{3} r_{3} \cosh \tilde{J} v+\left(1+\frac{\tilde{j}_{1}^{2}}{T^{2}} \hat{t} t\right) \cosh \frac{\tilde{J} v}{2}\right] \frac{c^{2} T^{2}}{\tilde{j}_{1}^{2}} \sinh \frac{\tilde{J} v}{2}\right\} .
\end{gathered}
$$

The left and right generators are connected by the relations

$$
\begin{array}{r}
\hat{r}_{1}=r_{1} \cosh \tilde{J} v+i r_{2} \sinh \tilde{J} v, \quad r_{2}=\hat{r}_{2} \cosh \tilde{J} v+i \hat{r}_{1} \sinh \tilde{J} v \\
r_{3}=\hat{r}_{3}\left(\cosh \tilde{J} v+i \frac{\tilde{j}_{1}}{T} t \sinh \tilde{J} v\right), t-\hat{t}=2 i \hat{r}_{3} r_{3} \frac{\tilde{j}_{1}}{c^{2} T} \sinh \frac{\tilde{J} v}{2} .
\end{array}
$$


For $\sigma_{I I}=(3,1,2,4,5)$ commutation relations of space and time generators of the (anti) de Sitter kinematics are

$$
\begin{gathered}
S_{v}^{4( \pm)}\left(\sigma_{I I}\right)=\left\{t, \mathbf{r} \mid \hat{r}_{1} t=\hat{t}\left(r_{1} \cosh \tilde{J} v+i r_{3} \sinh \tilde{J} v\right),\right. \\
\hat{t r}_{2}=\left(\hat{r}_{2} \cosh \tilde{J} v+i \frac{c T}{\tilde{j}_{1}} \sinh \tilde{J} v\right) t, \hat{t} r_{3}=\left(\hat{r}_{3} \cosh \tilde{J} v+i \hat{r}_{1} \sinh \tilde{J} v\right) t \\
\hat{r}_{1} r_{2}=\hat{r}_{2}\left(r_{1} \cosh \tilde{J} v+i r_{3} \sinh \tilde{J} v\right), \hat{r}_{2} r_{3}=\left(\hat{r}_{3} \cosh \tilde{J} v+i \hat{r}_{1} \sinh \tilde{J} v\right) r_{2}, \\
\left.\hat{r}_{1} r_{3}-\hat{r}_{3} r_{1}=2 i\left[\frac{\tilde{j}_{1}^{2}}{T^{2}} \hat{t} t \cosh \tilde{J} v+\left(1-\frac{\tilde{j}_{1}^{2}}{c^{2} T^{2}} \hat{r}_{2} r_{2}\right) \cosh \frac{\tilde{J} v}{2}\right] \frac{c^{2} T^{2}}{\tilde{j}_{1}^{2}} \sinh \frac{\tilde{J} v}{2}\right\} .
\end{gathered}
$$

The left and right generators are connected by the relations

$$
\begin{gathered}
\hat{r}_{1}=r_{1} \cosh \tilde{J} v+i r_{3} \sinh \tilde{J} v, \quad \hat{r}_{2}=r_{2} \cosh \tilde{J} v+2 \hat{t} t \tilde{j}_{1} \frac{c}{T} \sinh \frac{\tilde{J} v}{2}, \\
r_{3}=\hat{r}_{3} \cosh \tilde{J} v+i \hat{r}_{1} \sinh \tilde{J} v, \quad t=\hat{t} \cosh \tilde{J} v-\hat{t} r_{2} \frac{\tilde{j}_{1}}{c T} \sinh \tilde{J} v
\end{gathered}
$$

For $\sigma_{I I I}=(3,2,1,4,5)$ commutation relations of space and time generators of the (anti) de Sitter kinematics are

$$
\begin{gathered}
S_{v}^{4( \pm)}\left(\sigma_{I I I}\right)=\left\{t, \mathbf{r} \mid \hat{r}_{1} t=\hat{t}\left(r_{1} \cosh \tilde{J} v+i r_{3} \sinh \tilde{J} v\right),\right. \\
\hat{t} r_{2}-\hat{r}_{2} t=-2 \frac{c T^{2}}{\tilde{j}_{1}^{2}} \sinh \tilde{J} v, \hat{t} r_{3}=\left(\hat{r}_{3} \cosh \tilde{J} v+i \hat{r}_{1} \sinh \tilde{J} v\right) t, \\
\hat{r}_{1} r_{2}=\hat{r}_{2}\left(r_{1} \cosh \tilde{J} v+i r_{3} \sinh \tilde{J} v\right), \hat{r}_{2} r_{3}=\left(\hat{r}_{3} \cosh \tilde{J} v+i \hat{r}_{1} \sinh \tilde{J} v\right) r_{2}, \\
\left.\hat{r}_{1} r_{3}-\hat{r}_{3} r_{1}=2 i\left[\cosh \tilde{J} v+\frac{\tilde{j}_{1}^{2}}{T^{2}}\left(\hat{t} t-\frac{1}{c^{2}} \hat{r}_{2} r_{2}\right) \cosh \frac{\tilde{J} v}{2}\right] \frac{c^{2} T^{2}}{\tilde{j}_{1}^{2}} \sinh \frac{\tilde{J} v}{2}\right\} .
\end{gathered}
$$

The left and right generators are connected by the relations

$$
\begin{aligned}
& \hat{r}_{1}=r_{1} \cosh \tilde{J} v+i r_{3} \sinh \tilde{J} v, \quad r_{2}=\hat{r}_{2} \cosh \tilde{J} v+c \hat{t} \sinh \tilde{J} v \\
& r_{3}=\hat{r}_{3} \cosh \tilde{J} v+i \hat{r}_{1} \sinh \tilde{J} v, \quad \hat{t}=t \cosh \tilde{J} v-r_{2} \frac{1}{c} \sinh \tilde{J} v .
\end{aligned}
$$




\subsection{Quantum Minkowski kinematics}

Zero curvature limit $T \rightarrow \infty$ transform (anti) de Sitter kinematics to Minkowski one. For permutations $\hat{\sigma}, \tilde{\sigma}, \sigma_{I}, \sigma_{I I}, \sigma_{I I I}$ left and right space-time generators coincide $\hat{t}=t, \hat{r}_{k}=r_{k}$ and commutation relations take the form

$$
\begin{gathered}
M_{v}^{4}(\tilde{\sigma})=\left\{t, \mathbf{r} \mid\left[t, r_{p}\right]=0,\left[t, r_{3}\right]=-\frac{v}{c},\left[r_{1}, r_{2}\right]=-i v,\left[r_{p}, r_{3}\right]=0, p=1,2\right\}, \\
M_{v}^{4}(\hat{\sigma})=\left\{t, \mathbf{r} \mid\left[t, r_{p}\right]=0,\left[t, r_{3}\right]=i v,\left[r_{i}, r_{k}\right]=0, i, k=1,2,3\right\} \\
M_{v}^{4}\left(\sigma_{I}\right)=\left\{t, \mathbf{r} \mid\left[t, r_{k}\right]=0,\left[r_{1}, r_{3}\right]=\left[r_{2}, r_{3}\right]=0,\left[r_{1}, r_{2}\right]=i v\right\} \\
M_{v}^{4}\left(\sigma_{I I}\right)=\left\{t, \mathbf{r} \mid\left[t, r_{k}\right]=0,\left[r_{1}, r_{2}\right]=\left[r_{2}, r_{3}\right]=0,\left[r_{1}, r_{3}\right]=i v\right\} \\
M_{v}^{4}\left(\sigma_{I I I}\right)=\left\{t, \mathbf{r} \mid\left[t, r_{1}\right]=\left[t, r_{3}\right]=0,\left[t, r_{2}\right]=-\frac{v}{c}\right. \\
\left.\left[r_{1}, r_{2}\right]=\left[r_{2}, r_{3}\right]=0,\left[r_{1}, r_{3}\right]=i v\right\}
\end{gathered}
$$

For permutation $\check{\sigma}$ we have $\hat{t}=t, \hat{r}_{p}=r_{p}$, but $\hat{r}_{3}=r_{3}-\frac{v}{c} t^{2}$ and Minkowski kinematics is given by

$$
\begin{gathered}
M_{v}^{4}(\check{\sigma})=\left\{t, \mathbf{r} \mid\left[t, r_{p}\right]=0,\left[t, r_{3}\right]=\frac{v}{c} t\left(1-t^{2}\right),\left[r_{1}, r_{2}\right]=0,\right. \\
\left.\left[r_{2}, r_{3}\right]=\frac{v}{c} r_{2}\left(1-t^{2}\right),\left[r_{1}, r_{3}\right]=\frac{v}{c} r_{1}\left(1-t^{2}\right)\right\} .
\end{gathered}
$$

$M_{v}^{4}\left(\sigma_{I}\right)$ and $M_{v}^{4}\left(\sigma_{I I}\right)$ are connected by replacement of space generators $r_{2}$ with $r_{3}$ and vice-versa, therefore they can be regarded as equivalent kinematics. The same is true for $M_{v}^{4}(\tilde{\sigma})$ and $M_{v}^{4}\left(\sigma_{I I I}\right)$. so we obtain four nonequivalent Minkowski kinematics. 


\subsection{Quantum Newton kinematics}

Newton kinematics are obtained from (anti) de Sitter kinematics by the non-

relativistic limit $c \rightarrow \infty$ and are characterized by the following commutation relations of generators

$$
\begin{gathered}
N_{v}^{4( \pm)}(\tilde{\sigma})=\left\{t, \mathbf{r} \mid\left[t, r_{k}\right]=0, \quad\left[r_{1}, r_{2}\right]=-i v, \quad\left[r_{p}, r_{3}\right]=0\right\}, \\
N_{v}^{4( \pm)}(\check{\sigma})=\left\{t, \mathbf{r} \mid\left[t, r_{k}\right]=0, \quad\left[r_{1}, r_{2}\right]=i \tilde{j}_{1} \frac{v}{T} t^{2}, \quad\left[r_{p}, r_{3}\right]=0\right\}, \\
N_{v}^{4( \pm)}\left(\sigma_{I}\right)=\left\{t, \mathbf{r} \mid\left[t, r_{k}\right]=0,\left[r_{1}, r_{3}\right]=\left[r_{2}, r_{3}\right]=0,\left[r_{1}, r_{2}\right]=i v\left(1+\frac{\tilde{j}_{1}^{2}}{T^{2}} t^{2}\right)\right\}, \\
N_{v}^{4( \pm)}\left(\sigma_{I I}\right)=N_{v}^{4( \pm)}\left(\sigma_{I I I}\right)=\left\{t, \mathbf{r} \mid\left[t, r_{k}\right]=0,\left[r_{1}, r_{2}\right]=\left[r_{2}, r_{3}\right]=0\right. \\
\left.\left[r_{1}, r_{3}\right]=i v\left(1+\frac{\tilde{j}_{1}^{2}}{T^{2}} t^{2}\right)\right\} .
\end{gathered}
$$

Unlike previous cases, where $\hat{t}=t, \hat{r}_{k}=r_{k}$, for permutation $\hat{\sigma}$ the third left and right space generators are connected as $\hat{r}_{3}=r_{3}+i \tilde{j}_{1}^{2} \frac{v}{T^{2}}$, therefore commutation relations in this case are

$$
\begin{gathered}
N_{v}^{4( \pm)}(\hat{\sigma})=\left\{t, \mathbf{r} \mid\left[t, r_{p}\right]=0,\left[t, r_{3}\right]=i v\left(1+\frac{\tilde{j}_{1}^{2}}{T^{2}} t^{2}\right)\right. \\
\left.\left[r_{1}, r_{2}\right]=-i \tilde{j}_{1} \frac{v}{T} t r_{1},\left[r_{p}, r_{3}\right]=0\right\} .
\end{gathered}
$$

Nonequivalent (anti) de Sitter kinematics $S_{v}^{4( \pm)}\left(\sigma_{I I}\right)$ and $S_{v}^{4( \pm)}\left(\sigma_{I I I}\right)$ become identical one $N_{v}^{4( \pm)}\left(\sigma_{I I}\right)=N_{v}^{4( \pm)}\left(\sigma_{I I I}\right)$ in the non-relativistic limit. $N_{v}^{4}\left(\sigma_{I}\right)$ and $N_{v}^{4}\left(\sigma_{I I}\right)$ are connected by replacement of space generators $r_{2}$ with $r_{3}$ and vice-versa, therefore they can be regarded as equivalent kinematics. So there are four nonequivalent Newton kinematics.

\subsection{Quantum Galilei kinematics}

Galilei kinematics can be obtained by the non-relativistic limit $c \rightarrow \infty$ of the Minkowski kinematics (23), (24) or by the zero curvature limit $T \rightarrow \infty$ of 
the Newton kinematics $(\underline{25}),(26)$. They have identical left and right spacetime generators, which are commutative for $G_{v}^{4}(\check{\sigma})$. Galilei kinematics $G_{v}^{4}(\hat{\sigma})$ has one nonzero commutator of time and space generators $\left[t, r_{3}\right]=i v$ as well as Minkowski kinematics $M_{v}^{4}(\hat{\sigma})$ Minkowski kinematics $M_{v}^{4}(\hat{\sigma})(\underline{23})$. For Galilei kinematics $G_{v}^{4}(\tilde{\sigma}), G_{v}^{4}\left(\sigma_{I}\right), G_{v}^{4}\left(\sigma_{I I}\right), G_{v}^{4}\left(\sigma_{I I I}\right)$ there is only one nonzero commutator of space generators $\left[r_{1}, r_{2}\right]= \pm i v$ or $\left[r_{1}, r_{3}\right]=i v$, i.e. all these kinematics are physically equivalent. So there exist two nonequivalent noncommutative Galilei kinematics and for permutation $\check{\sigma}$ the non-relativistic and zero curvature limits give in result Galilei kinematics with totally commuting space-time generators.

\subsection{Quantum Carroll kinematics}

The exotic Carroll kinematics are also realized as constant curvature spaces for $j_{2}=j_{3}=1, j_{4}=\iota_{4}$, but with different interpretation of the Beltrami coordinates, namely $r_{k}=\xi_{k+1} \xi_{1}^{-1}, k=1,2,3$ are the space generators and $t=\xi_{5} \xi_{1}^{-1}$ is the time generator. Due to this interpretation the new physical dimensions of the contraction parameters appear: the parameter $j_{1}$ is replaced by $\tilde{j}_{1} R^{-1}$, where $R \rightarrow \infty$ corresponds to $j_{1}=\iota_{1}$ and $[R]=$ [length].

The nonequivalent Carroll kinematics with non-minimal multiplier $J=$ $j_{1}^{2} \iota_{4}$ are achieved for the three substitutions: $\hat{\sigma}=(2,1,3,4,5), \sigma^{\prime \prime}=(2,1,3,5,4)$, $\sigma^{\prime \prime \prime}=(2,3,1,5,4)$. The physical dimension of the deformation parameter is equal to $[v]=\left[R^{2}\right]=[\text { length }]^{2}$. The corresponding kinematics are given by space-time generators with the commutation relations

$$
C_{v}^{4( \pm)}(\hat{\sigma})=\left\{t, \mathbf{r} \mid\left[r_{1}, t\right]=i v\left(1+\frac{\tilde{j}_{1}^{2}}{R^{2}} \mathbf{r}^{2}\right),\left[t, r_{2}\right]=\left[t, r_{3}\right]=0,\left[r_{i}, r_{k}\right]=0\right\}
$$

where $\mathbf{r}^{2}=r_{1}^{2}+r_{2}^{2}+r_{3}^{2}$ with $\hat{r}_{k}=r_{k}$, but left and right time generators are connected as $\hat{t}=t+i \frac{\tilde{j}_{1}^{2}}{R^{2}} v r_{1}$;

$$
\begin{gathered}
C_{v}^{4( \pm)}\left(\sigma^{\prime \prime}\right)= \\
=\left\{t, \mathbf{r} \mid\left[t, r_{p}\right]=i v \frac{\tilde{j}_{1}^{3}}{R^{3}} r_{2}^{2} r_{p},\left[t, r_{2}\right]=i v \frac{\tilde{j}_{1}}{R}\left(1+\frac{\tilde{j}_{1}^{2}}{R^{2}} r_{2}^{2}\right) r_{2},\left[r_{i}, r_{k}\right]=0\right\},
\end{gathered}
$$


where $p=1,3, \hat{r}_{k}=r_{k}, \hat{t}=t-i \frac{\tilde{j}_{1}^{3}}{R^{3}} v r_{2}^{2}$ and

$$
\begin{gathered}
C_{v}^{4( \pm)}\left(\sigma^{\prime \prime \prime}\right)=\left\{t, \mathbf{r} \mid\left[t, r_{k}\right]=0,\left[r_{1}, r_{2}\right]=i v\left[1+\frac{\tilde{j}_{1}^{2}}{R^{2}}\left(t^{2}+r_{1}^{2}+r_{3}^{2}\right)\right],\right. \\
\left.\left[r_{1}, r_{3}\right]=\left[r_{2}, r_{3}\right]=0\right\},
\end{gathered}
$$

with $\hat{r}_{2}=r_{2}+i \frac{\tilde{j}_{1}^{2}}{R^{2}} v r_{1}, \hat{r}_{1}=r_{1}, \hat{r}_{3}=r_{3}, \hat{t}=t$. The kinematics $C_{v}^{4( \pm)}(\hat{\sigma})$ and $C_{v}^{4( \pm)}\left(\sigma^{\prime \prime \prime}\right)$ are transformed to each other by the replacement $t \rightarrow r_{2}, r_{2} \rightarrow t$, i.e. they are mathematically isomorphic, but physically nonequivalent. So we obtain tree nonequivalent Carroll kinematics.

Quantum analogs of the zero curvature Carroll kinematics are obtained in the limit $R \rightarrow \infty$. For $C_{v}^{4(0)}\left(\sigma^{\prime \prime}\right)$ all generators are commute. For $C_{v}^{4(0)}(\hat{\sigma})$ the only nonzero commutator is $\left[r_{1}, t\right]=i v$ and for $C_{v}^{4(0)}\left(\sigma^{\prime \prime \prime}\right)$ such commutator is $\left[r_{1}, r_{2}\right]=i v$.

\section{Conclusion}

The quantum Cayley-Klein spaces of constant curvature $O_{q}^{N}(j ; \sigma)$ are uniformly obtained from the quantum Euclidean space $O_{q}^{N}$ in Cartesian coordinates by the standard trick with real, complex, and nilpotent numbers, using a q-analog of Beltrami coordinates. The transformation of the quantum deformation parameter $Z=J v$ under contraction is the important ingredient of the noncommutative quantum groups and noncommutative quantum spaces. Unlike previous papers on this subject [1, 2] second power of contraction parameters are included in the multiplier $J$, what make all kinematics contractions admissible. The different combinations of quantum structure and Cayley-Klein scheme of contractions and analytical continuations are described with the help of permutations $\sigma$. As a result the quantum deformations of four dimensional (anti) de Sitter, Minkowski, Newton, Galilei and Carroll kinematics with non-minimal transformations of deformation parameter are obtained.

We have found six types of the noncommutative realistic (anti) de Sitter kinematics with non-minimal multipliers, which admit all non-relativistic and zero curvature limits and three types of the nonequivalent Carroll kinematics. For Minkowski and Newton kinematics there are four types and two types for noncommutative Galilei kinematics. In addition every kinematics has two 
types with minimal multipliers [1]. This demonstrate a wide variety of the quantum deformations of the commutative space-time models. The remarkable property of the limit kinematics is that for some of them commutation relations of generators are proportional to a numbers instead of generators, for example, all Minkowski (23), Newton $N_{v}^{4( \pm)}(\tilde{\sigma})(\underline{25})$, all Galilei and all flat Carroll kinematics. Moreover Galilei $G_{v}^{4}(\check{\sigma})$ and Carroll $C_{v}^{4(0)}\left(\sigma^{\prime \prime}\right)$ kinematics have commutative space-time generators. In other words commutative Galilei kinematics or simplest deformations of Galilei and Minkowski kinematics, when zero commutation relations of generators become proportional to a numbers, can be obtain from quantum (anti) de Sitter kinematics by contractions with non-minimal transformations of deformation parameter.

The quantum Galilei kinematics $G_{v}^{4}(\tilde{\sigma}), G_{v}^{4}\left(\sigma_{I}\right), G_{v}^{4}\left(\sigma_{I I}\right), G_{v}^{4}\left(\sigma_{I I I}\right)$, Newton $N_{v}^{4( \pm)}(\tilde{\sigma})$, Minkowski $M_{v}^{4}\left(\sigma_{I}\right)$ and Carroll $C_{v}^{4(0)}\left(\sigma^{\prime \prime \prime}\right)$ have the same nonzero commutator $\left[r_{1}, r_{2}\right]=i v$. Equal nonzero commutator $\left[t, r_{3}\right]=i v$ have Minkowski $M_{v}^{4}(\hat{\sigma})$, Galilei $G_{v}^{4}(\hat{\sigma})$ and Carroll $C_{v}^{4(0)}(\hat{\sigma})$ kinematics. In spite of the fact that commutation relations of generators of some kinematics are identical, these kinematics are physically different. Mathematically isomorphic kinematics may be physically nonequivalent.

\section{Acknowledgments}

This work was supported by the program "Fundamental problems of nonlinear dynamics" of the Russian Academy of Sciences.

\section{References}

[1] N. A. Gromov and V. V. Kuratov, J. Math. Phys. 47, 013502 (2006).,

[2] N. A. Gromov and V. V. Kuratov, hep-th/0410086.

[3] H. Bacry and J.-M. Levy-Leblond, J. Math. Phys. 9, 1605 (1968).

[4] L. D. Faddeev, N. Yu. Reshetikhin and L. A. Takhtajan, Algebra i Analis 1, 178 (1989), (in Russian).

[5] E. Inön $\ddot{u}$ and E. P. Wigner, Proc. Nat. Acad. Sci. USA 39, 510 (1953). 